\title{
Induced anisotropies in ferromagnetic amorphous ribbons locally annealed by laser
}

\author{
C. Aroca and M. C. Sánchez \\ Departamento Física Materiales, Facultad de Ciencias Fisica, Universidad Complutense, Madrid 28040, Spain \\ I. Tanarro \\ Instituto de Estructura de la Materia, Consejo Superior de Investigaciones Cientificas, Madrid, Spain \\ P. Sánchez \\ Departamento de Fisica, Escuela Técnica Superior de Ingenenieros Telecomunicación \\ Universidad Politecnica, Madrid 28040, Spain \\ E. López \\ Departamento de Física Materiales, Facultad de Ciencias Fisica, Universidad Complutense, Madrid 28040, Spain \\ M. Vázquez \\ Departamento de Ingenenieros Civil, E.U.I.T., Obras Publicas, Universidad Politecnica, Madrid, Spain \\ (Received 26 March 1990)
}

\begin{abstract}
The origin of anisotropy induced by local laser annealing has been studied in amorphous ferromagnetic materials with different magnetostriction constants. The results have been explained using a theoretical model based on the assumption that the origin of the induced anisotropies are internal stresses produced by inhomogeneous heat flows during the local laser annealing. The induced anisotropies are not uniform, which produces a magnetostatic energy that can modify the induced magnetoelastic anisotropy. This effect can be used to induce different anisotropies by changing the shape of the local laser annealing.
\end{abstract}

\section{INTRODUCTION}

The magnetic behavior of ferromagnetic amorphous samples can be modified by inducing anisotropies through stress or field annealing. The possible origin of these anisotropies in the creation of a local order in the sample. ${ }^{1-3}$

Recently, a high-power laser has been used as heat source to anneal amorphous ferromagnetic materials in two ways: (a) by heating the sample uniformly, which induces anisotropies with a similar mechanism to that of the conventional annealing; ${ }^{4-6}$ (b) by producing inhomogeneous heat flows in the sample, it can induce higher anisotropies that can be due to the apparition of internal stresses. $^{7-9}$ This latter technique can be also used to crystallize the sample locally, this produces interesting effects. $^{5}$

The effect of the inhomogeneous laser annealing has been studied in samples locally irradiated by a laser, to produce geometrical arrays of annealed zones. The work has been performed by measuring the magnetization curves, induced anisotropy, and Bitter pattern. The results are discussed from a model based in the stresses induced by the reduction of volume in the annealed areas, and by the magnetostatic energy due to the different magnetic permeabilities of the annealed and unannealed zones.

\section{ANISOTROPY MEASUREMENT}

The samples were locally irradiated for $0.5 \mathrm{~s}$, through a continuous 5-W Ar laser focused by a suitable micro- scope objective to obtain a spot size of $10 \mu \mathrm{m}$. Different arrays were induced, with different symmetries, one regular with distances between annealed zones $1.2 \times 1.2 \mathrm{~mm}^{2}$ (fourfold symmetry) and an irregular one with $0.6 \times 2.4$ $\mathrm{mm}^{2}$ (twofold symmetry) (Fig. 1). Measurements were performed in samples with high-magnetostriction constant (Metglas 2605SC) and in a low-magnetostriction one (Metglas 2705M). The samples were cut, by electrical abrasion, in form of disk in order to avoid the shape factor.

The as-cast sample $2705 \mathrm{M}$ shows an anisotropy perpendicular to the rolling direction (as can be seen in Fig.

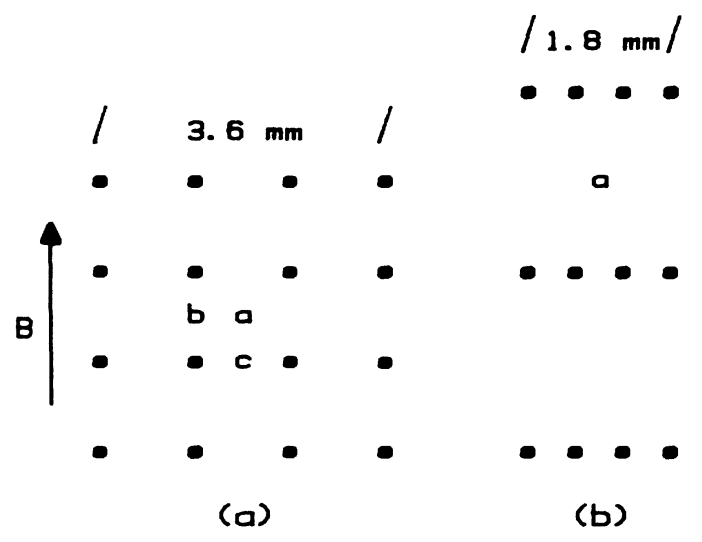

FIG. 1. Schematic of different annealing distribution: (a) fourfold, (b) twofold. 


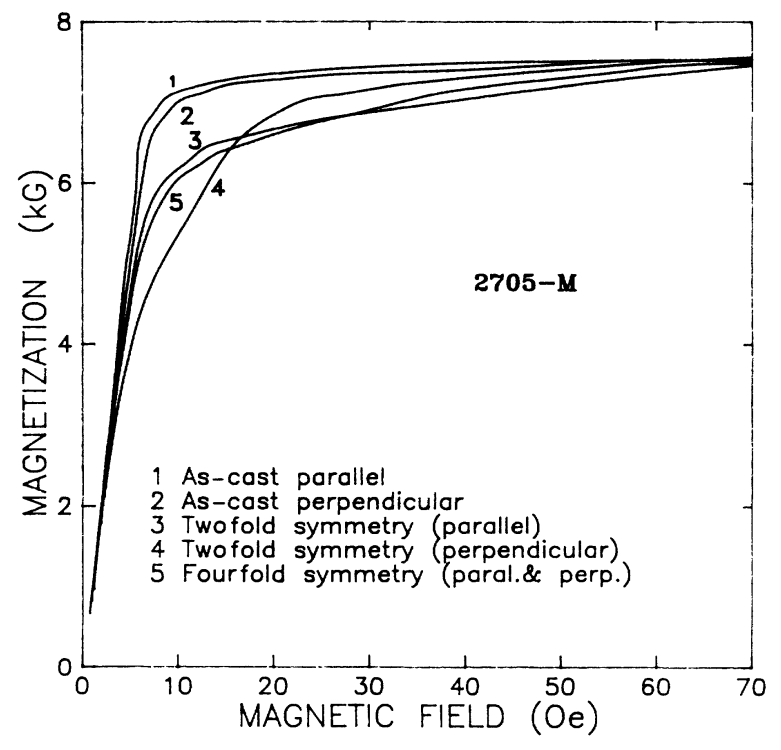

FIG. 2. Magnetization curves, parallel and perpendicular to the rolling directions and to the impact rows. Sample 2705M.

2). At low magnetic field, the $2605 \mathrm{SC}$ sample presents the same magnetization energy in parallel and perpendicular directions to the rolling, but at high field the magnetization energy depends on the direction of the applied field (Fig. 3). It suggests the existence of areas with perpendicular anisotropy to the surface of the sample, that can be only observed at high fields. ${ }^{10}$ The domain observation by Bitter technique shows strips domains, that confirms this assumption.

The twofold symmetry locally annealed samples show, for both samples, an in plane anisotropy, parallel or perpendicular to the rows of annealed zones, depending on the magnetostriction constant $\lambda_{s}$ of the sample. The in-

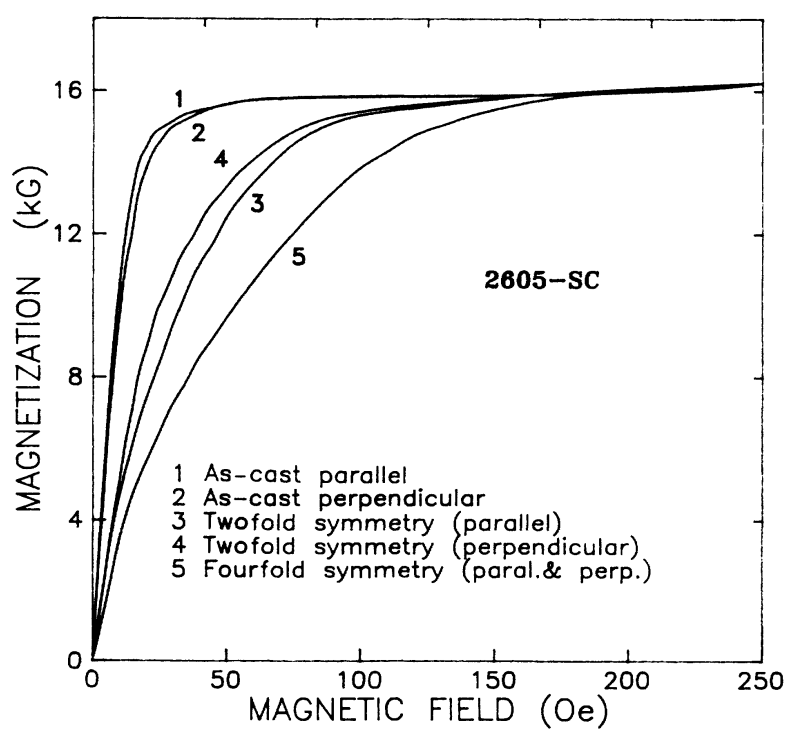

FIG. 3. Magnetization curves, parallel and perpendicular to the rolling directions and to the impact rows. Sample $2605 \mathrm{SC}$.

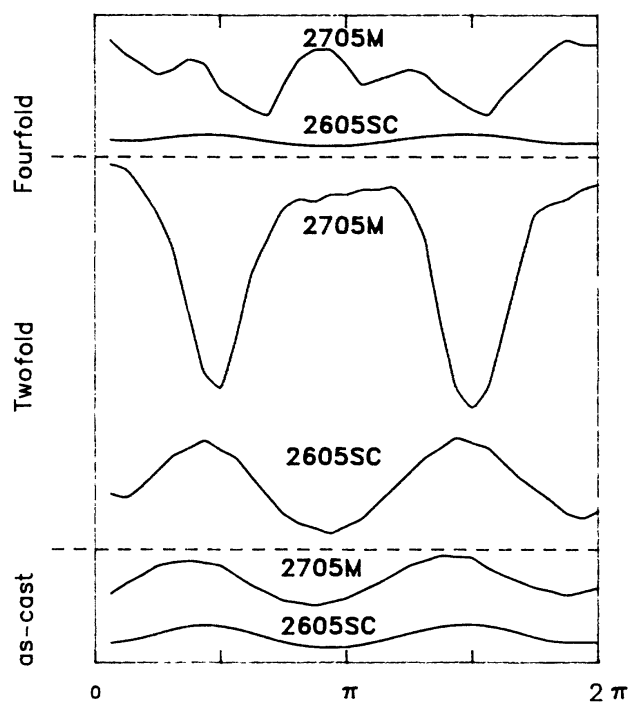

FIG. 4. $(M, \phi)_{H}$ curves for the two samples.

plane-induced anisotropy, in the low $\lambda_{s}$, is higher than in the 2605SC one, and the permeability decreases in both samples, being this effect stronger in the 2605SC.

The magnetization energy for the fourfold symmetry locally annealed samples is the same in both direction and in both kinds of samples. Figure 4 shows the curves of maxima magnetization versus the angle between the rolling direction and the applied field $H$, at a constant amplitude of $H$. The maxima of these curves correspond to the maxima distance between the curves of magnetization, parallel and perpendicular to the easy axis. These curves show that the $2605 \mathrm{SC}$ annealed sample is almost isotropic, whereas in the $2705 \mathrm{M}$ samples a biaxial anisotropy is induced.

\section{STRESS MODEL}

The anisotropies induced by laser irradiation can be due to the tensile stresses created by the change of volume in the annealed zones. As a consequence of the laser irradiation, the impacted zones become crystallized and change their volume. ${ }^{9}$ It produces a nonuniform stresses distribution, that can be calculated.

Taking the origin of the reference system in the center of the laser impact, the internal stresses in an unlimited free sample, by symmetry consideration, are

$$
\begin{aligned}
& \sigma_{r}=S / r^{2}+2 C, \\
& \sigma_{\theta}=-S / r^{2}+2 C,
\end{aligned}
$$

where $S$ and $C$ are constants, and $r$ and $\theta$ polar coordinates.

By applying the boundary conditions

$$
\sigma_{r}=\sigma_{\theta}=0 \text { for } r=\infty \text {. }
$$

If $r_{0}$ is the radius of the area to be irradiated and $\Delta r_{0}$ is the change that experiments this radius after the laser ir- 


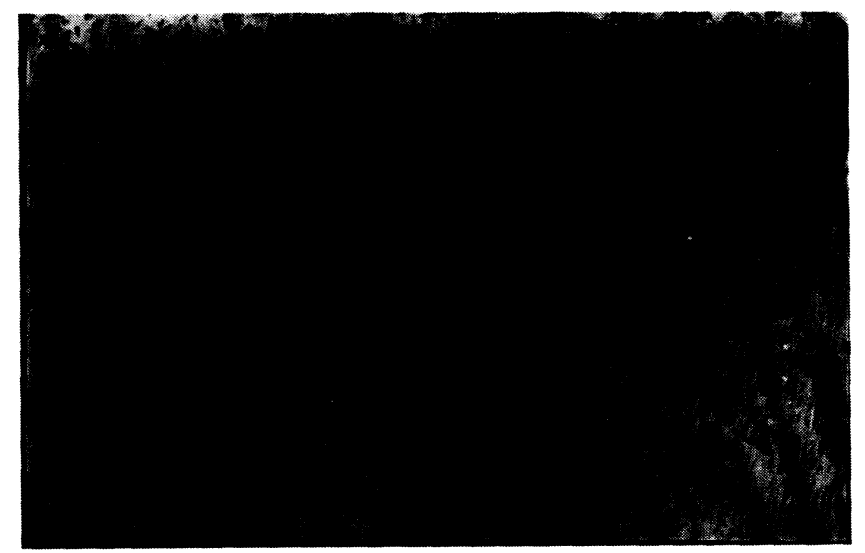

FIG. 5. Bittern pattern in a $\lambda_{s}>0$ sample with a single laser impact. The DW's are in radial directions.

radiation, taking into account that

$$
\Delta r_{0}=\int_{r_{0}}^{\infty} \epsilon_{r} d r
$$

where $\epsilon_{r}$ is the strain in the $r$ direction, the internal stresses can be written as

$$
\begin{aligned}
& \sigma_{r}=\left[E \rho_{0} /(1+v)\right]\left(r_{0}^{2} / r^{2}\right), \\
& \sigma_{\theta}=-\left[E \rho_{0} /(1+v)\right]\left(r_{0}^{2} / r^{2}\right),
\end{aligned}
$$

being $E$ the Young modulus, $v$ the Poisson modulus, and $\rho_{0}=\Delta r_{0} / r_{0}$.

These stresses, tensile and compressive, induce an anisotropy of energy:

$$
K_{r}=-3 \lambda_{s} \sigma_{r} \cos ^{2} \alpha \cos ^{2} \beta,
$$

being $\alpha$ the angle between the in-plane magnetization with the radial direction, and $\beta$ the angle between the magnetization and the surface of the sample.

From these results, in $\lambda_{s}>0$ samples the magnetization and the domain walls (DW's) will be along the radial directions, whereas in $\lambda_{s}<0$ samples the magnetization, and the DW's, will be in circles around the laser impact. Experimentally, by Bitter technique, we have observed

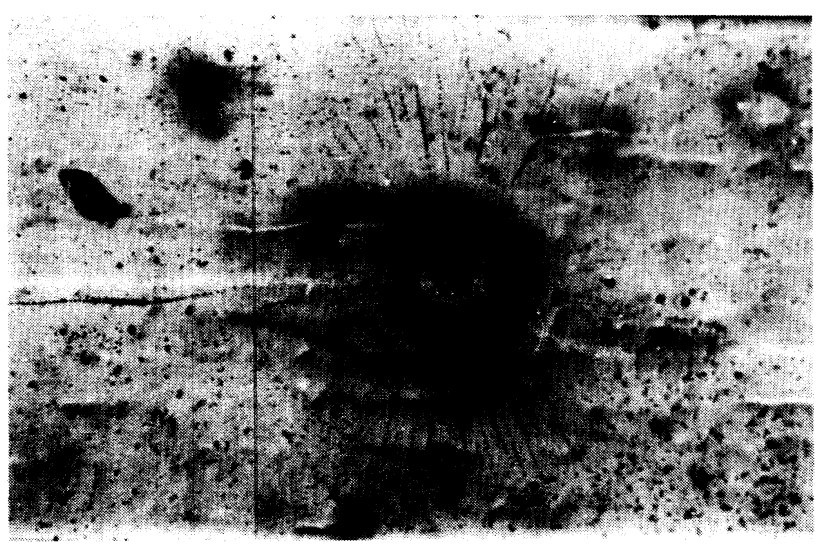

FIG. 6. Bittern pattern in a $\lambda_{s}<0$ sample with a single laser impact. The DW's are surrounding the laser impact.

these domains and DW's in both kinds of samples. In Fig. 5, $\lambda_{s}>0$, the DW's in radial directions can be seen. In Fig. $6, \lambda_{s}<0$, the DW's are circles surrounding the impact.

Inside the $r<r_{0}$ zone, there are uniform tensile stresses in all directions,

$$
\sigma_{r}=\sigma_{\theta}=E \rho_{0} /(1+v),
$$

that induce an anisotropy energy

$$
K_{r}=-\left(\frac{3}{2}\right) \lambda_{s} \sigma_{s} \cos ^{2} \beta .
$$

So, if $\lambda_{s}>0$, an easy plane parallel to the surface of the sample in the annealed zones will be induced. If $\lambda_{s}<0$, an easy axis perpendicular to the surface in the annealed zone will be induced and therefore stripe domains surrounding the impact will appear. The Bitter patterns are in good agreement with these assumptions, as can be seen in Fig. 6.

In the case of an array of annealed areas, the induced stresses are the addition of the ones produced by each laser impact. If the laser impacts are separated by a distance, $a$ in the $X$ direction, and $b$ in the $Y$ direction, the stresses in the point $(x, y)$ are:

$$
\begin{aligned}
& \sigma_{x}=-\sigma_{y}=A \sum_{i=-n}^{n} \sum_{j=-n}^{n}\left\{\left[(a i-x)^{2}-(b j-y)^{2}\right] /\left[(a i-x)^{2}+(b j-y)^{2}\right]^{2}\right\}, \\
& \tau_{x y}=2 A \sum_{i=-n}^{n} \sum_{j=-n}^{n}\left\{-(a i-x)(b j-y) /\left[(a i-x)^{2}+(b j-y)^{2}\right]^{2}\right\},
\end{aligned}
$$

being $A=E r_{0}^{2} \Delta r_{0} /\left[(1+v) r_{0}\right], \sigma_{x}$ and $\sigma_{y}$ the internal stresses in the $X$ and $Y$ direction, and $\tau_{x y}$ the shear stress.

Figure 7 represents the tensile stress field, obtained by computer, in samples locally annealed with fourfold [Fig. 8(a)] and twofold [Fig. 8(b)] symmetries. The compressive lines will be perpendicular to the tensile lines. In the positive $\lambda_{s}$ samples, both the anisotropy, and the magnetization, will be parallel to the tensile stress lines, whereas in the negative $\lambda_{s}$ samples, they will follow the compressive stress lines. In the fourfold symmetry locally annealed samples, in the equidistant point between the impacts, the influence of the stresses due to the impacts is

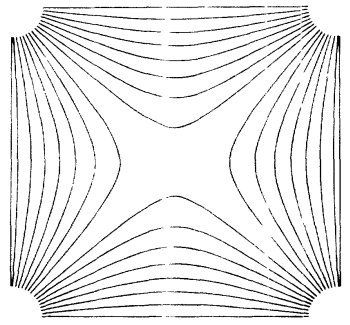

(a)

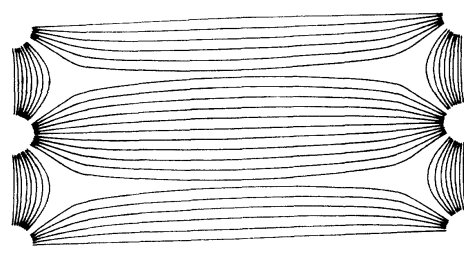

(b)
FIG. 7. Tensile stress lines obtained by computer for (a) fourfold and (b) twofold symmetry locally annealed samples. 

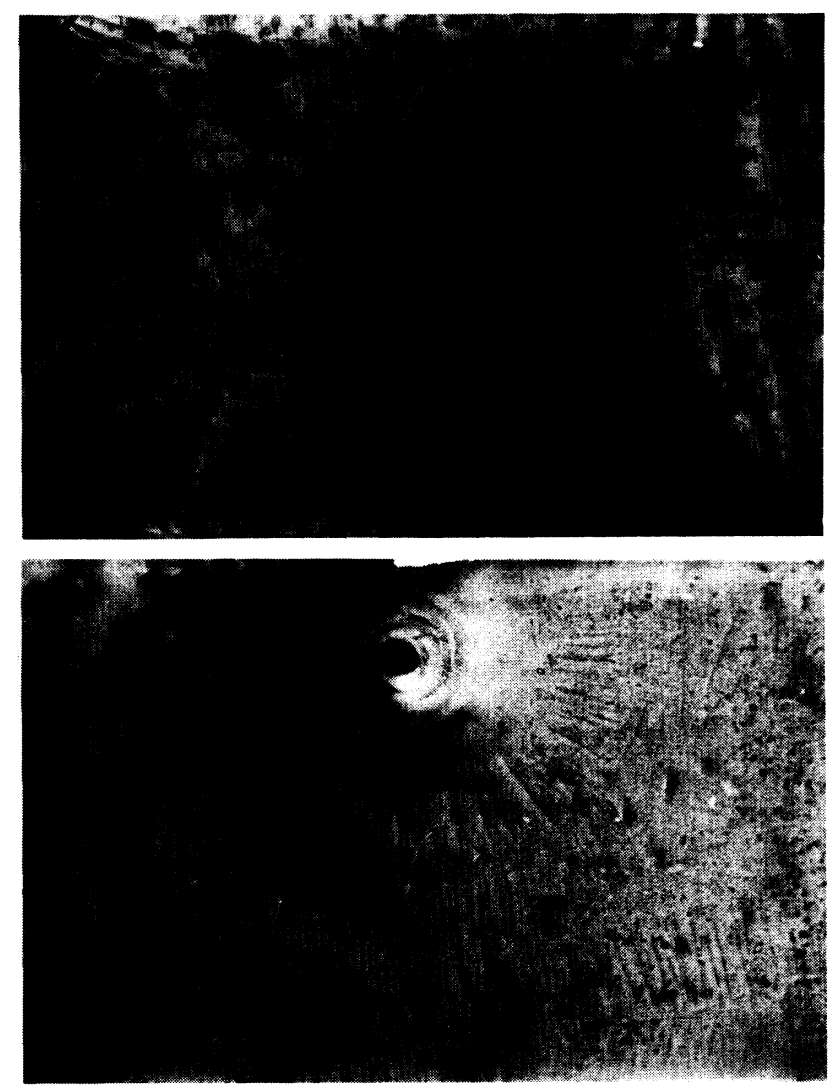

FIG. 8. Bittern pattern in (a) $\lambda_{s}>0$ and (b) $\lambda_{s}<0$ samples with fourfold symmetry locally annealed.
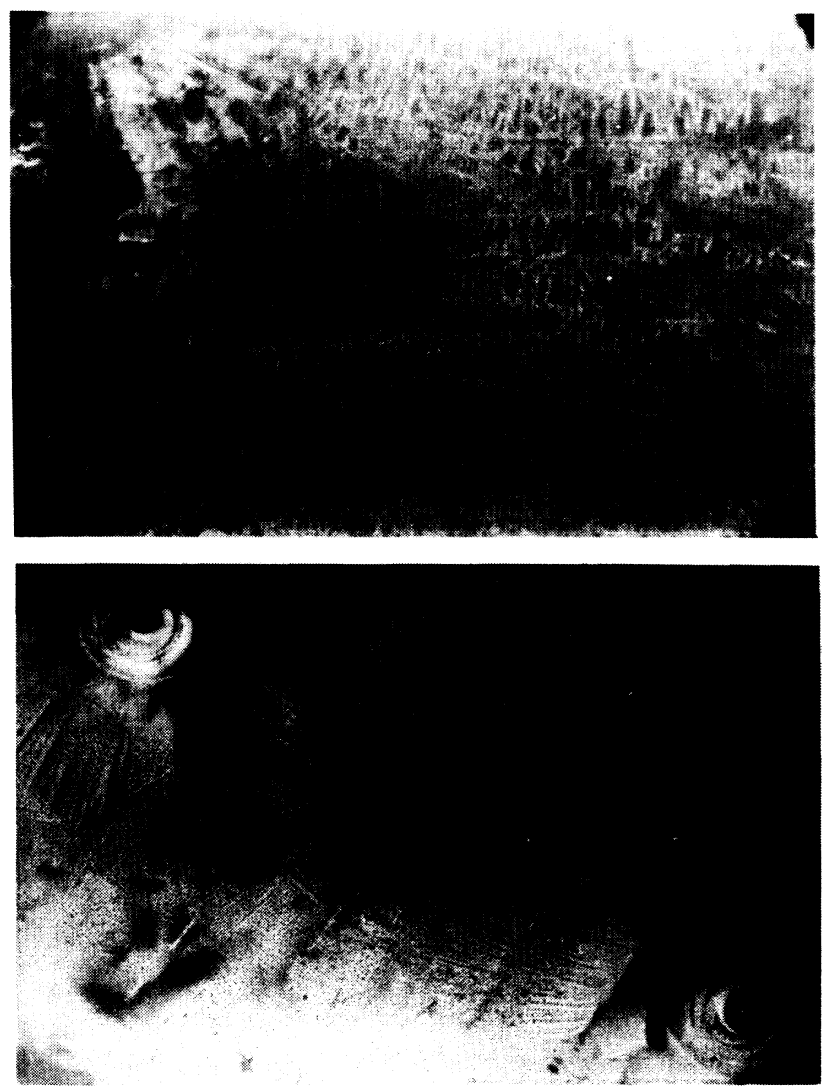

FIG. 9. Bittern pattern in (a) $\lambda_{s}>0$ and (b) $\lambda_{s}<0$ samples with twofold symmetry locally annealed. negligible, and so the anisotropy will be the initial anisotropy in the samples. Figures $8(a)$ and $8(b)$ show the Bitter pattern of a fourfold symmetry annealed samples with positive and negative magnetostriction. Figures 9(a) and 9(b) show the same for twofold symmetry annealed samples. Once more it is evident that the results are in agreement with the calculations.

Using as a light source, a very stabilized He-Ne laser, we have obtained hysteresis loops, by magneto-optic transversal Kerr effect, in small areas of the samples (200 $\mu \mathrm{m})$. The hysteresis loops confirm the results shown above. ${ }^{11}$

\section{ANISOTROPY MEASUREMENT AND DOMAIN CONFIGURATION}

By comparing the anisotropy measurements and Bitter pattern we can deduce the following consequences.

(a) Fourfold symmetry locally annealed samples. Samples with $\lambda_{s}<0$ exhibit an in-plane biaxial anisotropy in agreement with the calculations performed above and with the Bitter pattern. The anisotropy measurements show that $\lambda_{s}>0$ samples are in-plane isotropic (Fig. 4). This result disagrees with the calculations and domain observations. It can be explained taking into account that, in the 2605SC samples, the DW's end in the zones impacted by laser, with very low permeability due to its crystallization. Any domain-wall displacement will increase the magnetostatic energy, reducing strongly the permeability in the zones where the magnetization processes are due to DW displacement. Besides, the DW's are placed in zones with a high-stress gradient, that pin the DW's reducing also the permeability. Consequently, there is almost the same difficulty to displace DW's than to rotate the magnetization.

In the case of $\lambda_{s}<0$ samples, the DW's are surrounding the rows of the laser impacts, then the magnetostatic effects do not appear. Besides the stress gradient is very small due to the low $-\lambda_{s}$ value, and so the DW's will move almost freely.

(b) Twofold symmetry locally annealed samples. The anisotropy measurements and Bittern pattern observations are in agreement, except in the value of the induced anisotropy. Samples with higher magnetostriction should show higher-anisotropy energy. However, in our results the anisotropy energy of the 2705SC sample is lower than the anisotropy energy of the $2705 \mathrm{M}$ sample. It can be explained if we take into account again that the easy axis is perpendicular to the rows of the laser impacts (Fig. 1). The easy axis due to the magnetostatic energy is perpendicular to the easy axis due to the internal stresses, so it reduces the total-anisotropy energy of the sample. In the 2075M sample both easy axis have the same direction and the total anisotropy energy will be higher than in 2605SC sample, in spite of its magnetostriction values.

\section{CONCLUSIONS}

In this work we have shown it is possible to induce, in a simple and predictable way, very different anisotropies in samples with negative magnetostriction. The direction 
as well as the energy of the induced anisotropy can be changed by an adequate local laser annealing from a point to other of the sample. Then it is possible the tailoring of a material for a certain application.

In positive magnetostriction samples, in principle, also is possible to induce any kind of anisotropies by local laser annealing, however, the anisotropy due to mag- netostatic effect is opposite to the magnetoelastic one and so the effects are lowers.

\section{ACKNOWLEDGMENTS}

This work has been partially supported by the Comisión Interministerial de Ciencia y Tecnologia (CIDCYT) (No. Mat88-0646-C03).
1J. M. Riveiro, G. Rivero, and M. C. Sanchez, J. Magn. Magn. Mater. 31-34, 1551 (1983).

${ }^{2}$ F. E. Luborsky, J. J. Becker, and R. O. McCary, IEEE Trans. Magn. MAG-11, 1644 (1975).

${ }^{3}$ J. M. Riveiro, M. C. Sanchez, and C. Aroca, Ann. Fisica B 80, 27 (1984).

${ }^{4}$ L. Lanotte and J. Phys. Lett. 44, 541 (1983).

${ }^{5}$ M. Garcia and M. C. Sanchez, Rev. Phys. Appl. 21, 207 (1986).

${ }^{6}$ L. Lanotte and V. Tagliaferri, High Temp. Mat. Proc. 7, 25 (1986).
${ }^{7}$ S. Tsukahara, Y. Yocoyama, T. Tanaka, and A. Kokubu, IEEE Trans. Magn. MAG-20, 51308 (1984).

${ }^{8}$ P. Sanchez, M. C. Sanchez, E. Lopez, M. Garcia, and C. Aroca, J. Phys. (Paris) Colloq. 12, T49, C8-1325 (1988).

${ }^{9}$ C. Aroca, M. C. Sanchez, I. Tanarro, E. Lopez, and P. Sanchez, J. Magn. Magn. Mater. 83, 349 (1990).

${ }^{10}$ P. Sanchez, E. Lopez, M. C. Sanchez, and C. Aroca, J. Magn. Magn. Mater. 75, 233 (1988).

${ }^{11}$ M. C. Sanchez, I. Tanarro, M. Vazquez, M. Barradi, E. Lopez, P. Sanchez, and C. Aroca (unpublished). 


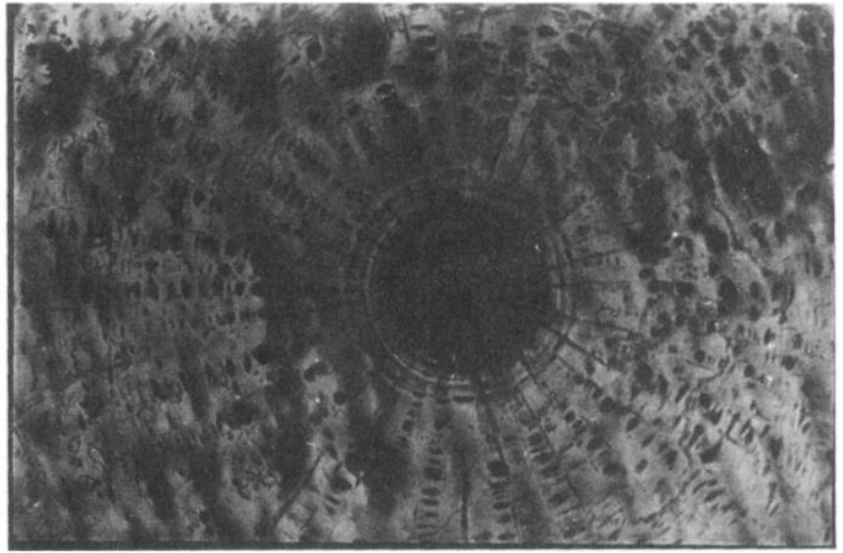

FIG. 5. Bittern pattern in a $\lambda_{s}>0$ sample with a single laser impact. The DW's are in radial directions. 


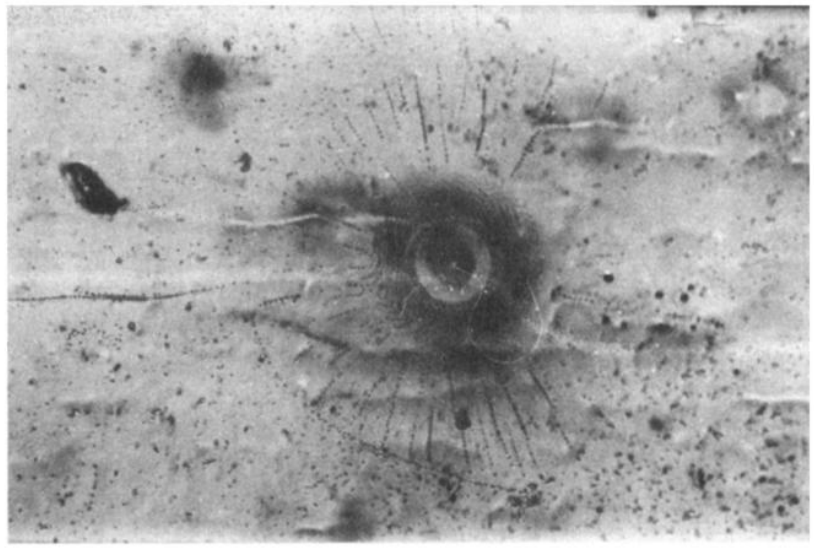

FIG. 6. Bittern pattern in a $\lambda_{s}<0$ sample with a single laser impact. The DW's are surrounding the laser impact. 

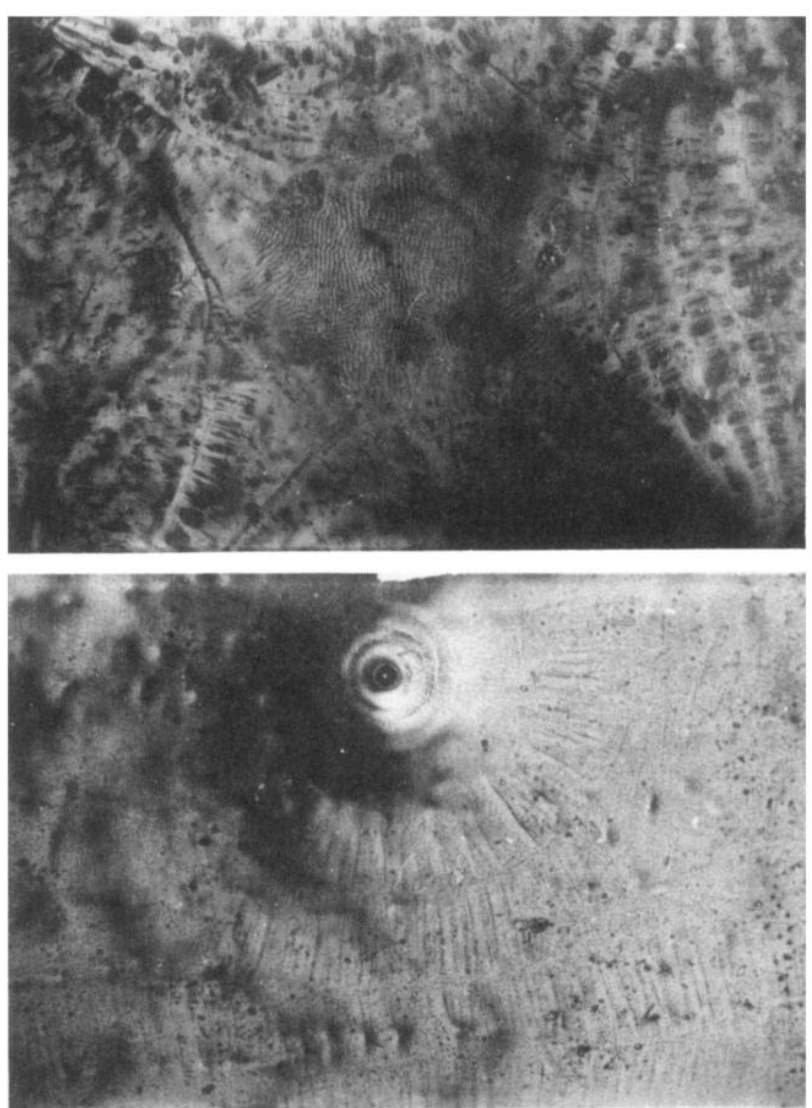

FIG. 8. Bittern pattern in (a) $\lambda_{s}>0$ and (b) $\lambda_{s}<0$ samples with fourfold symmetry locally annealed. 

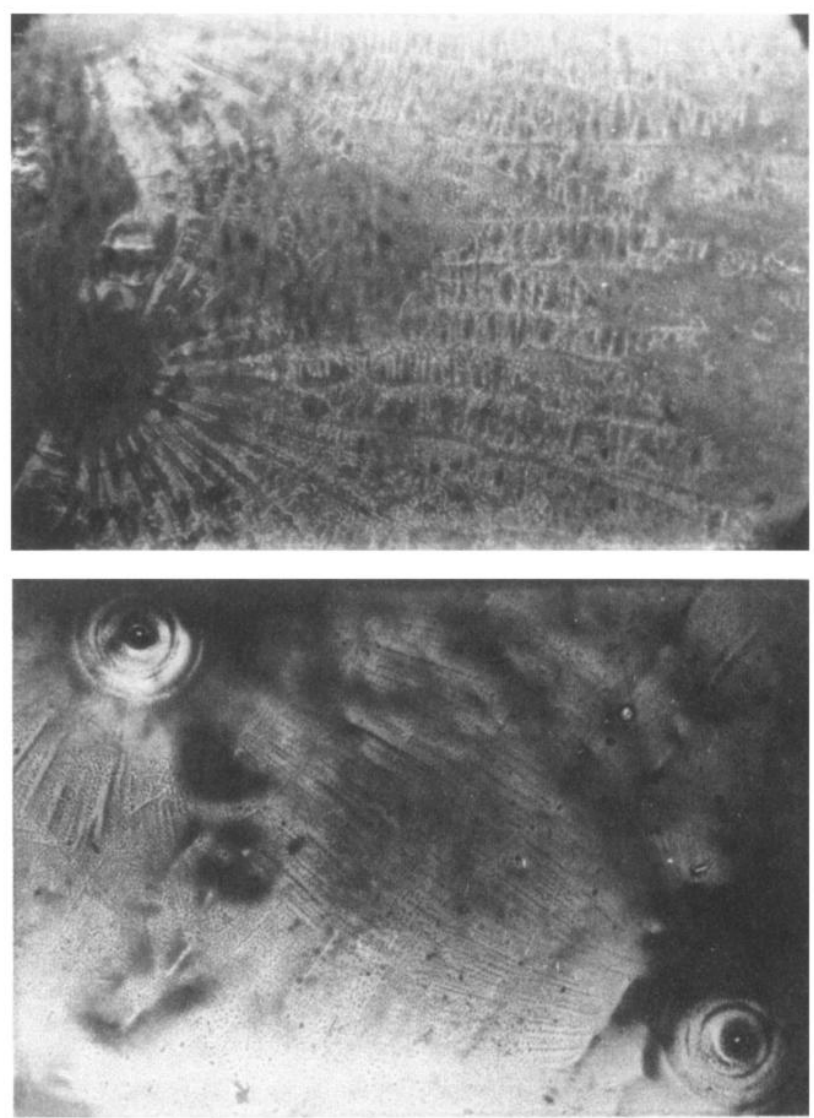

FIG. 9. Bittern pattern in (a) $\lambda_{s}>0$ and (b) $\lambda_{s}<0$ samples with twofold symmetry locally annealed. 